\title{
Large-eddy simulation of conductive flows at low magnetic Reynolds number
}

\author{
By B. Knaepen AND P. Moin
}

\section{Introduction}

In large-eddy simulations (LES), only the large-scale structures of the flow are simulated directly while the small-scale structures are taken into account through a model, referred to as the subgrid-scale (SGS) model. This results in significant reduction in computational requirements. The trade-off comes in the extra modelling effort that has to be produced in order to adequately take into account the discarded small scales structures. In the computational fluid mechanics community, the most widely used SGS model is the Smagorinsky eddy viscosity model (Smagorinsky 1963). This model has gained an even bigger practical interest following the work of Germano et al. (1991) in which the dynamic procedure was introduced. The dynamic procedure enables optimization "on the fly" of the arbitrary scaling factor that is inherently present in the original Smagorinsky model (more details below) and thus allows the model to automatically adapt to the flow being studied.

In this paper we study the LES method with dynamic procedure in the context of conductive flows subject to an applied external magnetic field at low magnetic Reynolds number $R_{m}$. These kind of flows are encountered in many industrial applications. For example, in the steel industry, applied magnetic fields can be used to damp turbulence in the casting process. In nuclear fusion devices (Tokamaks), liquid-lithium flows are used as coolant blankets and interact with the surrounding magnetic field that drives and confines the fusion plasma. Also, in experimental facilities investigating the dynamo effect, the flow consists of liquid-sodium for which the Prandtl number and, as a consequence, the magnetic Reynolds number is low.

Most of the previous works considering LES in the case of MHD flows have been directed towards flows at high $R_{m}$, where the full non-linear MHD equations have to be used, or towards flows in the absence of an external magnetic field: Yoshizawa (1987); Zhou \& Vahala (1991); Theobald et al. (1994); Agullo et al. (2001); Müller \& Carati (2002). To our knowledge, the only attempt to study MHD turbulence at low magnetic Reynolds number from the LES point of view is due to Shimomura (1991). In that work, the author considers the case of magnetohydrodynamic turbulent channel flow and introduces a new SGS model designed to incorporate the effects of the applied uniform magnetic field. However, like the original Smagorinsky model, the model of Shimomura contains constants that have to be adjusted and are thus flow dependent. In the present paper, we show that it is possible to circumvent this problem by the use of the dynamic Smagorinsky model.

Our attention is focused here on the case of homogeneous (initially isotropic) decaying turbulence. The numerical simulations performed mimic the thought experiment described in Moffatt (1967) in which an initially homogeneous isotropic conductive flow is suddenly subjected to an applied magnetic field and freely decays without any forcing. Note that this flow was first studied numerically by Schumann (1976). It is well known 
that in that case, extra damping of turbulence occurs due to the Joule effect and that the flow tends to become progressively independent of the coordinate along the direction of the magnetic field. Our comparison of filtered direct numerical simulation (DNS) predictions and LES predictions show that the dynamic Smagorinsky model enables one to capture successfully the flow with LES, and that it automatically incorporates the effect of the magnetic field on the turbulence.

Our paper is organized as follows. In the next section we summarize the LES approach in the case of MHD turbulence at low $R_{m}$ and recall the definition of the dynamic Smagorinsky model. In Sec. 3 we describe the parameters of the numerical experiments performed and the code used. Section 4 is devoted to the comparison of filtered DNS results and LES results. Conclusions are presented in Sec. 5.

\section{LES at low magnetic Reynolds number}

For homogeneous flows, the magnetic Reynolds number can be defined through the following relation:

$$
R_{m}=\frac{u L}{\eta}
$$

where $u=\sqrt{\left\langle u_{i} u_{i}\right\rangle / 3}$ is the r.m.s. of the fluctuating velocity $u_{i}, L$ is the integral length scale of the flow and $\eta$ is the magnetic diffusivity. $R_{m}$ represents the relative importance of the non-linear terms and the diffusion term in the magnetic induction equation. In the limit of low $R_{m}$, the MHD equations can be simplified considerably Roberts (1967). It is indeed possible to close independently the momentum equation and take into account the effect of the magnetic field through an extra damping term. This approximation is known as the quasi-static (QS) approximation and along with the incompressibility condition, $\partial_{i} u_{i}=0$, reads,

$$
\partial_{t} u_{i}=-\partial_{i}(p / \rho)-u_{j} \partial_{j} u_{i}-\frac{\left(B_{z}^{e x t}\right)^{2}}{\eta} \Delta^{-1} \partial_{z} \partial_{z} u_{i}+\nu \Delta u_{i}
$$

where $p$ is the sum of the kinematic and magnetic pressures, $\rho$ is the fluid density, $\nu$ the kinematic viscosity, $B_{z}^{e x t}$ is the applied external magnetic field and $\Delta^{-1}$ is the inverse of the Laplacian operator. Note that $B_{z}^{e x t}$ has by convention been aligned with the $z$ direction and expressed in Alfven units.

LES equations are obtained by filtering (2.2). The filtered velocity $\bar{u}_{i}$ is defined by,

$$
\bar{u}_{i}(\mathbf{x})=\int G(\mathbf{x}, \mathbf{y}) u_{i}(\mathbf{y}) d \mathbf{y}
$$

where $G$ is a smoothing kernel that eliminates the small scale part of $u_{i}$ and satisfies the relation,

$$
\int G(\mathbf{x}, \mathbf{y}) d \mathbf{y}=1 .
$$

In terms of the filtered velocity, Eq. 2.2 can be written as,

$$
\partial_{t} \bar{u}_{i}=-\partial_{i}(\bar{p} / \rho)-\overline{\bar{u}_{j} \partial_{j} \bar{u}_{i}}-\frac{\left(B_{z}^{e x t}\right)^{2}}{\eta} \Delta^{-1} \partial_{z} \partial_{z} \bar{u}_{i}+\nu \Delta \bar{u}_{i}-\partial_{j} \bar{\tau}_{i j},
$$


where,

$$
\bar{\tau}_{i j}=\overline{u_{i} u_{j}}-\overline{\bar{u}_{i} \bar{u}_{j}},
$$

is the subgrid-scale stress tensor. In order to close $(2.5), \bar{\tau}_{i j}$ has to be expressed only in terms of the filtered velocity.

Is is interesting to note that $\bar{\tau}_{i j}$ does not depend explicitly on the magnetic field. Indeed, the magnetic contribution, being a linear term in (2.2), does not require an explicit SGS counterpart in the LES equation. In the case of non-conductive flows, the most widely used model for $\bar{\tau}_{i j}$ is the Smagorinsky model:

$$
\bar{\tau}_{i j}=-2 C_{s} \bar{\Delta}^{2}\left|\bar{S}_{\mid}\right| \bar{S}_{i j}, \quad \bar{S}_{i j}=\frac{1}{2}\left(\partial_{i} \bar{u}_{j}+\partial_{j} \bar{u}_{i}\right), \quad \bar{S}=\sqrt{2 \bar{S}_{i j} \bar{S}_{i j}},
$$

where $\bar{\Delta}$ is the width of the filter $G$ and $C_{s}$ is the Smagorinsky constant. However, as was observed by Shimonura in Shimomura (1991), model (2.7) with $C_{s}$ optimized for a non-conductive flow, is not adequate in the present context. This is easily understood when one refers to the fact that the applied external magnetic field has the tendency to suppress non-linear transfers in the velocity field. Thus, the effect of the SGS stress tensor needs to be decreased for conductive flows when the external magnetic field is switched on. This should result in a lower optimal value for the Smagorinsky constant.

Contrary to what was done in Shimomura (1991), we will not explicitly modify the Smagorinsky model to incorporate the effect of the magnetic field but rather we will make use of the dynamic procedure (Germano et al. 1991) to optimize the value of $C_{s}$.

To define the dynamic procedure one introduces a second, coarser filter called the test-filter which we denote by $\approx$. Applying this filter in addition to $(2.5)$ yields,

$$
\partial_{t} \widetilde{\bar{u}}_{i}=-\partial_{i}(\tilde{\bar{p}} / \rho)-\widetilde{\widetilde{\bar{u}_{j} \partial_{j} \widetilde{\bar{u}}_{i}}}-\frac{\left(B_{z}^{e x t}\right)^{2}}{\eta} \Delta^{-1} \partial_{z} \partial_{z} \widetilde{\bar{u}}_{i}+\nu \Delta \widetilde{\bar{u}}_{i}-\partial_{j} \widetilde{\bar{\tau}}_{i j}-\partial_{j} \widetilde{\bar{L}}_{i j}
$$

where $\widetilde{\bar{L}}_{i j}=\widetilde{\overline{\bar{u}}_{i} \bar{u}_{j}}-\widetilde{\widetilde{\bar{u}}_{i} \widetilde{\bar{u}}_{j}}$ is the Leonard tensor which does not require any modeling since it is expressed in closed form using the filtered velocity $\bar{u}_{i}$. The sum $\widetilde{\bar{\tau}}_{i j}+\widetilde{\bar{L}}_{i j}$ represents the SGS stress tensor $\widetilde{\bar{T}}_{i j}$ of the combined $\stackrel{\sim}{\cdots}$ filter and we have the well-known Germano identity,

$$
\widetilde{\bar{L}}_{i j}=\widetilde{\bar{T}}_{i j}-\widetilde{\bar{\tau}}_{i j}
$$

Assuming that $\bar{u}_{i}$ and $\widetilde{\bar{u}}_{i}$ are self-similar, a suitable model for $\widetilde{\bar{T}}_{i j}$ should be,

$$
\widetilde{\bar{T}}_{i j}=-2 C_{s} \widetilde{\bar{\Delta}}^{2} \mid \widetilde{\bar{S}}^{\mid} \widetilde{\bar{S}}_{i j}, \quad \widetilde{\bar{S}}_{i j}=\frac{1}{2}\left(\partial_{i} \widetilde{\bar{u}}_{j}+\partial_{j} \widetilde{\bar{u}}_{i}\right), \quad \widetilde{\bar{S}}=\sqrt{2 \widetilde{\bar{S}}_{i j} \widetilde{\bar{S}}_{i j}},
$$

where $\tilde{\bar{\Delta}}$ is the width of the $\stackrel{\sim}{\simeq}$ filter. When one models $\bar{\tau}_{i j}$ and $\widetilde{\bar{T}}_{i j}$ using $(2.7)$ and (2.10), the Germano identity will undoubtedly be violated. However, the constant $C_{s}$ can be chosen in such a way as to minimize the difference (in the least square sense) between both sides of (2.9). Assuming homogeneity in all directions, the optimal choice is (Lilly 1992):

$$
C_{s}=\frac{\left\langle\widetilde{\bar{M}}_{i j} \widetilde{\bar{L}}_{i j}\right\rangle}{\left\langle\widetilde{\bar{M}}_{i j} \widetilde{\bar{M}}_{i j}\right\rangle}, \quad \widetilde{\bar{M}}_{i j}=2\left[\bar{\Delta}^{2}\left|{\widetilde{S} \mid \bar{S}_{i j}}-\widetilde{\bar{\Delta}}^{2}\right| \widetilde{\bar{S}} \mid \widetilde{\bar{S}}_{i j}\right]
$$


where $\langle\cdots\rangle$ denotes spatial averaging. Thus, although model (2.7) does not explicitly incorporate the magnetic field, the dynamic constant $C_{s}$ should adjust to an appropriate value if the scale-similarity hypothesis is at all justified.

\section{Numerical experiments}

To assess the dynamic Smagorinsky model in the present context, we have built a set of DNS databases. Since we restrict our attention to a cubic domain with periodic boundary conditions, a spectral (dealiased) code has been used.

The velocity field can then be initialized in Fourier space. The initial mode amplitudes are computed to match the spectra of the Comte-Bellot \& Corrsin (1971) experiment at stage 1 (see Rogallo (1981) for more information on the procedure). Phases are initially random and the flow is left to freely decay until the skewness of the velocity derivative reaches a quasi constant value of $S=-0.4$. At this time (hereafter referred to as $t_{0}$ ) the flow is considered "physical" and used as the initial condition for all of our runs. All the DNS simulations are done in a $(2 \pi)^{3}$ box using a resolution of $512^{3}$ Fourier modes and the viscosity is set to $\nu=0.006$. Other relevant quantities measured at $t=t_{0}$ are summarized here:

$$
\begin{aligned}
R e & =\frac{u L}{\nu}=380 \quad \text { (integral Reynolds number) } \\
R_{\lambda} & =\sqrt{\frac{15}{\epsilon \nu}} u^{2}=84.1 \quad \text { (microscale Reynolds number), } \\
\tau_{e d d y} & =\frac{3}{2} \frac{u^{2}}{\epsilon}=0.238 \quad \text { (eddy turnover time). }
\end{aligned}
$$

Three test cases have been considered. The first one corresponds to a decaying flow without the addition of any applied external magnetic field. The other two numerical experiments are distinguished by their characteristic interaction numbers at $t_{0}$. The interaction number $N$ (also known as the Stuart number) is defined as follows:

$$
N=\frac{\left(B_{z}^{e x t}\right)^{2}}{\eta} \frac{L}{u}
$$

$N$ measures the relative strengths of the magnetic damping term and the non-linear term in (2.2). Two cases are examined here, $N=1$ and $N=10$.

To illustrate the effect of the external magnetic field, the time history of the kinetic energy density $E=\frac{1}{V} \int d \mathbf{x} \frac{1}{2} u_{i}(\mathbf{x}) u_{i}(\mathbf{x})$ of the flow for the three cases is presented in Fig. 1. As in all the following figures, time has been normalized by the initial eddy turnover time and the non-dimensional time is denoted by $t^{*}$.

\section{LES results}

In order to assess the LES method, some DNS snapshots of the flow field have been truncated from the $512^{3}$ resolution to a $32^{3}$ resolution (using a sharp Fourier cut-off). The initial condition for the LES runs has been obtained in the same way by truncating the DNS field at $t=t_{0}$. As can be easily seen from (2.11), the only free parameter in the subgrid-scale model is the ratio $\tilde{\bar{\Delta}} / \bar{\Delta}$. As is standard practice in LES of non-conductive flows, the value of 2 is adopted here. 


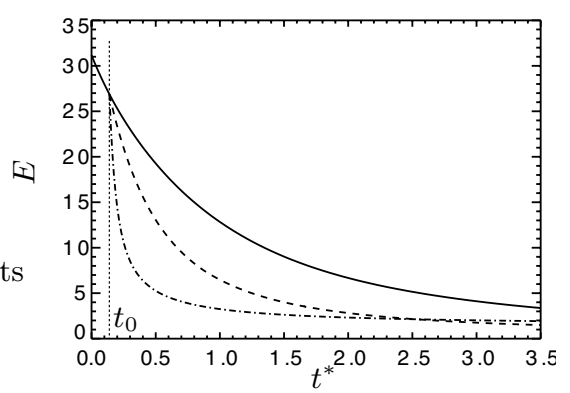

Figure 1. Time history of the kinetic energy density $E$. The solid line represents the flow decay without applied magnetic field, the dashed curve corresponds to the case $N=1$ and the dash-dot curve corresponds to the case $N=10$. The dotted line represents the time $t_{0}$ at which the magnetic field is switched on.
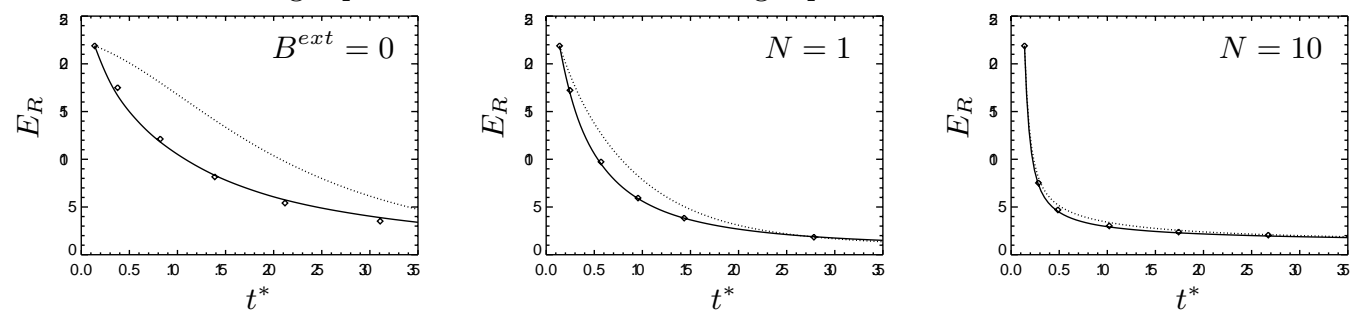

FIgURE 2. Time history of the resolved kinetic energy density: LES vs. filtered DNS. The solid lines represent the predictions of the LES. The diamonds represent the filtered kinetic energy density obtained by truncating the DNS fields to a $32^{3}$ resolution. The dotted lines represent the predictions of the unresolved DNS (performed on the $32^{3}$ mesh) without any subgrid-scale model.

\subsection{Kinetic Energy}

Figure 2 represents the time evolution of the resolved kinetic energy density $E_{R}=$ $\frac{1}{V} \int d \mathbf{x} \frac{1}{2} \bar{u}_{i}(\mathbf{x}) \bar{u}_{i}(\mathbf{x})$ predicted by the LES and compared to the filtered DNS. On each plot, a third curve representing a simulation (referred to as 'unresolved DNS') on the $32^{3}$ mesh without SGS modeling is added to stress the action of the subgrid-scale model. The case $B^{e x t}=0$ serves as a benchmark to check that in the case of non-conductive flows, our LES code behaves as expected. In both the cases $N=1$ and $N=10$, the LES performs remarkably well. In the case $N=1$ the difference between LES and unresolved DNS is very clear. In the case $N=10$ and for this diagnostic, the unresolved DNS does not depart significantly from the filtered DNS and LES.

\subsection{Energy spectra}

The energy spectrum constitutes a finer diagnostic than the kinetic energy density since it retains information about the repartition of energy among the different scales of the flow. Figures 3 and 4 contain respectively kinetic energy spectra for the cases $N=1$ and $N=10$ at several instants in the simulation. Agreement between LES and DNS is again excellent. The unresolved DNS exhibits the usual pile up of energy at high wave numbers that results from lack of resolution. Thus, although in terms of global energy the unresolved DNS does perform quite well in the $N=10$ case, the spectral properties of the flow are poorly predicted and the LES does a much better job. 

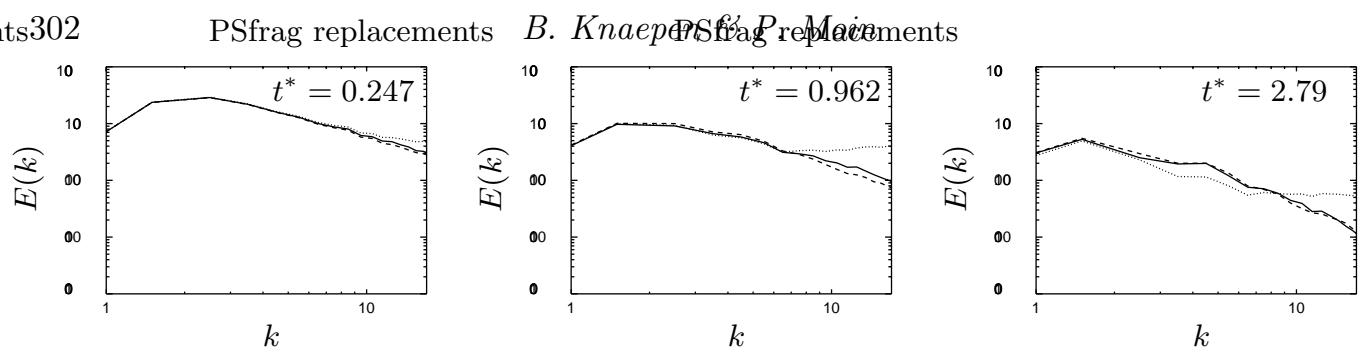

FiguRE 3 . Three-dimensional kinetic energy spectra for the case $N=1$. The solid line represents the filtered DNS, the dashed line represents the LES and the dotted line corresponds to the unresolved DNS. The times at which the spectra are calculated are indicated in the plots.
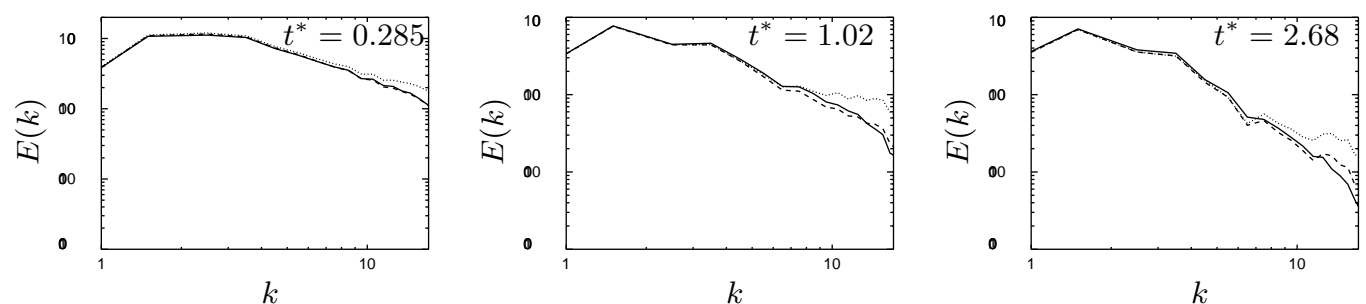

FiguRE 4 . Three-dimensional kinetic energy spectra for the case $N=10$. The solid line represents the filtered DNS, the dashed line represents the LES and the dotted line corresponds to the unresolved DNS. The times at which the spectra are calculated are indicated in the plots.

\subsection{Dissipation rate budget}

The evolution of the resolved kinetic energy density $E_{R}$ is governed by,

$$
\frac{d E_{R}}{d t}=-\frac{1}{V} \int d \mathbf{x}\left(\epsilon_{\nu}+\epsilon_{B}+\epsilon_{s g s}\right)
$$

where,

$$
\epsilon_{\nu}=2 \nu \bar{S}_{i j} \bar{S}_{i j}, \quad \epsilon_{B}=\frac{\left(B_{z}^{e x t}\right)^{2}}{\eta} \bar{u}_{i} \Delta^{-1} \partial_{z} \partial_{z} \bar{u}_{i}, \quad \epsilon_{s g s}=\bar{\tau}_{i j} \bar{S}_{i j}
$$

From Fig. 2 it is clear that the LES predicts the total dissipation rate very well. It is however instructive to know how the different contributions (4.2) are reproduced separately.

In Fig. 5 we present the time evolution of the resolved viscous dissipation rate $\epsilon_{\nu}$ for the LES, the filtered DNS and the unresolved DNS. The figures show that the LES reproduces this diagnostic very well, whereas the unresolved DNS systematically overestimates it. This results from the pile-up of energy near the LES cut-off where most of the resolved viscous dissipation occurs.

Figure 6 represents the time evolution of the resolved magnetic dissipation $\epsilon_{B}$. Again the LES predictions match the filtered DNS results very well. The differences with the unresolved DNS are less severe than for the viscous dissipation. This is to be expected since the magnetic dissipation occurs at every scale in the flow and its overall intensity is thus less contaminated by the pile-up of energy occurring near the LES cut-off for the unresolved DNS. It is also interesting to note that in the case $N=10$, the magnetic dissipation falls very rapidly with time at the early stages of the decay. This happens because in that case the flow quickly becomes fairly independent of the $z$-direction which is parallel to the magnetic field (this is illustrated further in Section 4.4).

Finally, in Fig. 7 the evolution of the subgrid-scale transfer rate $\epsilon_{s g s}$ is presented. For 

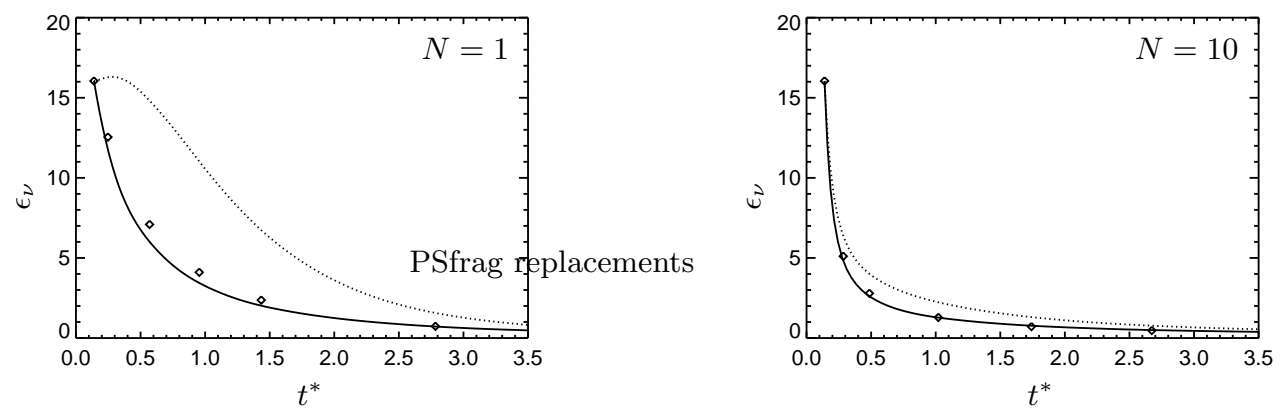

Figure 5. Resolved viscous dissipation rate $\epsilon_{\nu}$ for the cases $N=1$ (left) and $N=10$ (right). In each plot, the solid line represents the LES, the diamonds represent the filtered DNS and the dotted line represents the unresolved DNS.
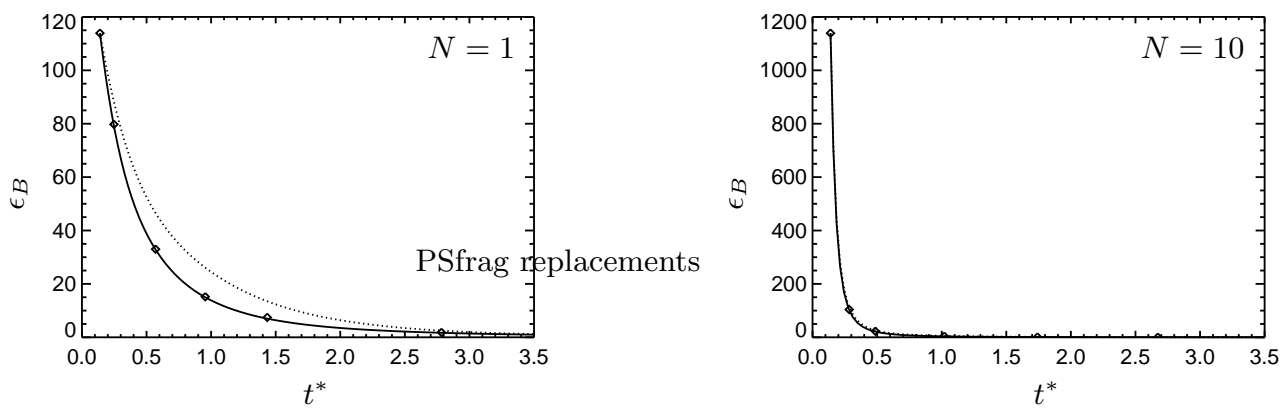

Figure 6 . Resolved magnetic dissipation rate $\epsilon_{B}$ for the cases $N=1$ (left) and $N=10$ (right). In each plot, the solid line represents the LES, the diamonds represent the filtered DNS and the dotted line represents the unresolved DNS.

the LES, $\epsilon_{s g s}$ is obtained by substituting the dynamic Smagorsinky in place of $\bar{\tau}_{i j}$ in (4.2). DNS results are obtained by computing the exact $\bar{\tau}_{i j}=\overline{u_{i} u_{j}}-\overline{\bar{u}}_{i} \bar{u}_{j}$ from the DNS data fields. Obviously, no (non-zero) results are available for the unresolved DNS. From the plots we see that the subgrid-scale transfer rate is initially overestimated in the LES. This is not a surprise since the dynamic procedure usually needs a little time to settle. Soon after this short transient time, the agreement between LES and DNS predictions is very good.

\subsection{Flow structures}

It is well known that the extra damping term present in the quasi-static approximation (2.2) leads to a progressive suppression of spatial variations in the flow along the direction of the magnetic field. It is thus important to assess how well the LES is able to reproduce this feature. To that end, we have plotted in Figures 8 and 9 the contours of the kinetic energy density at three different times respectively for the filtered DNS and the LES (only the case $N=10$ is shown because the effect is more pronounced for strong applied magnetic fields). Since the turbulence is decaying with time, the colormap had to be rescaled for each of the single plots. However, the same colormap for the filtered DNS and LES has been used in the plots corresponding to the same times. As is obvious from the plots, the LES reproduces the filtered DNS structures very well. The unresolved DNS also captures the large-scales structures of the flow (no plots shown) but due to the pile 

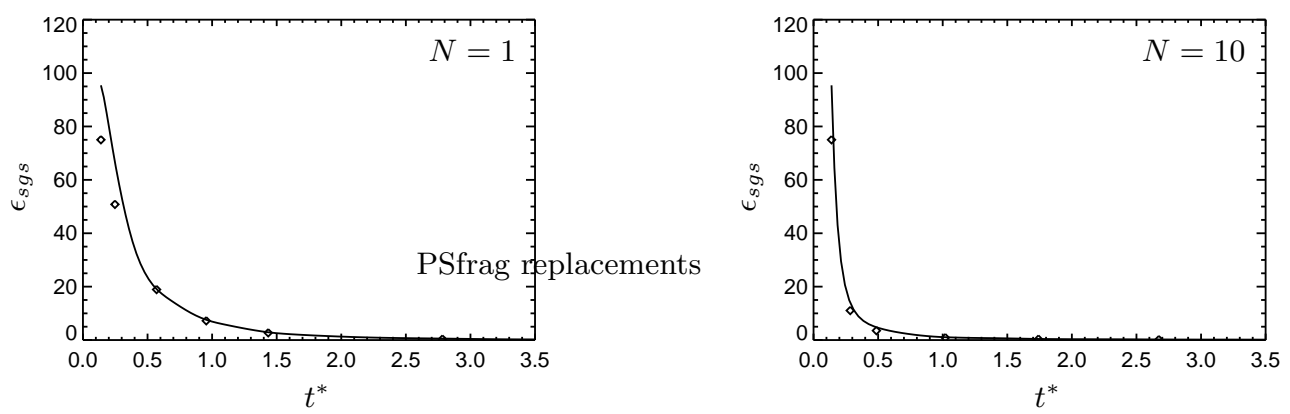

FiguRE 7 . Subgrid-scale transfer rate $\epsilon_{\text {sgs }}$ for the cases $N=1$ (left) and $N=10$ (right). In each plot, the solid line represents results obtained from the LES while the diamonds are obtained from the DNS data.
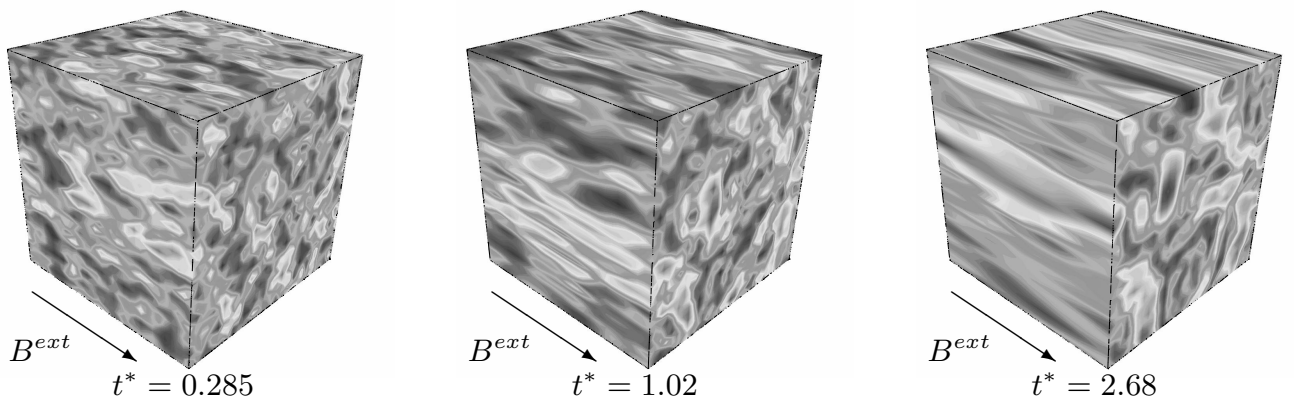

Figure 8 . Contours of the kinetic energy obtained from the filtered DNS $(N=10$ case). The different times at which the contours are calculated are indicated under the plots and are the same ones as those used in Fig. 9.
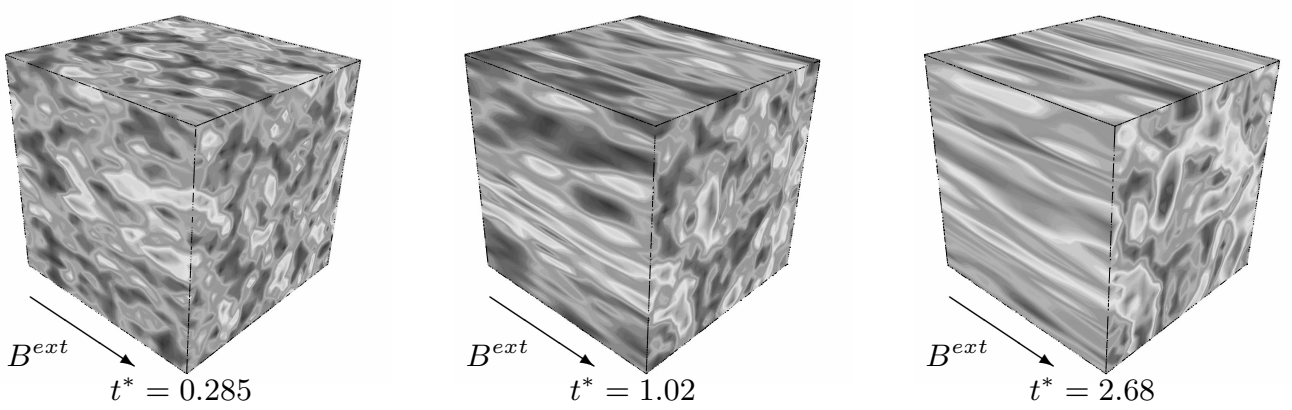

Figure 9. Contours of the kinetic energy obtained from the LES ( $N=10$ case). The different times at which the contours are calculated are indicated under the plots and are the same ones as those used in Fig. 8.

up of energy it predicts a significant amount of small scales; the corresponding contour plots contain some excessive 'noise' on top of the large-scales structures.

\subsection{Dynamic Constant}

As recalled in Section 2, the dynamic procedure is designed to optimize the scaling constant $C_{s}$ present in the Smagorinsky subgrid-scale model. We also mentioned that when an external magnetic field is present, the value of $C_{s}$ should decrease since nonlinear transfers are reduced and the effect of the subgrid-scale model should be damped 


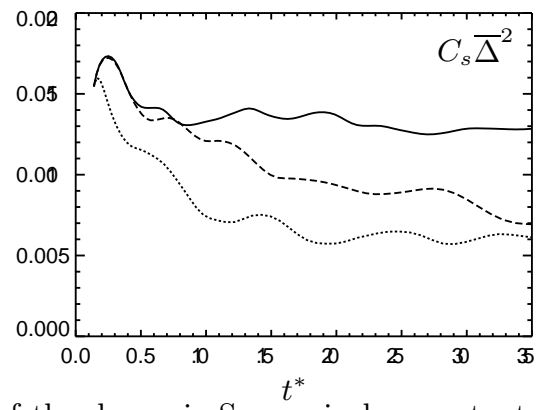

Figure 10. Time history of the dynamic Smagorinsky constant. The solid line represents the case with no magnetic field, the dashed line corresponds to the case $N=1$ and the case $N=10$ is represented by the dotted line.

accordingly. In Fig. 10 the evolution of $C_{s} \bar{\Delta}^{2}$ is plotted. From the figure, it is clear that the expected behavior is observed. As the interaction number is increased, the dynamic procedure automatically adapts the value of the Smagorinsky constant to decrease the effect of the subgrid-scale model.

\section{Conclusions}

In this article we have shown that the dynamic Smagorinsky model can be used to perform large-eddy simulations of flows subject to an applied external magnetic field at low magnetic Reynolds number, $R_{m}$. Although this subgrid-scale model was not designed for this application, its behavior is excellent owing to its adaptation to the flow and the applied magnetic field through the dynamic procedure. The model can be considered robust since it works equally well for interaction (or Stuart) numbers ranging from $N=0$ (no magnetic field) to $N=10$ (for which the flow becomes nearly two dimensional). In the future, the same model will be tested in more complex geometries.

\section{Acknowledgments}

The authors are grateful to O. Debliquy, Y. Dubief, M. Fatica, A. Honein, S. Kassinos for fruitful discussions and to G. Burton for his comments on this manuscript.

\section{REFERENCES}

Agullo, O., Muller, W.-C., Knaepen, B. \& Carati, D. 2001 Large eddy simulation for decaying magnetohydrodynamics turbulence with dynamic subgrid modeling. Phys. Plasmas 7, 3502-3505.

Comte-Bellot, G. \& Corrsin, S. 1971 Simple eulerian time correlations of full- and narrow-band velocity signals in grid generated 'isotropic' turbulence. J. Fluid Mech. 48, 273-337.

Germano, M., Piomelli, U., Moin, P. \& Cabot, W. 1991 A dynamic subgrid-scale eddy-viscosity model. Phys. Fluids A 3 (7), 1760-1765.

LiLLY, D. 1992 A proposed modification of the germano subgrid-scale closure method. Phys. Fluids 4, 633-635.

Moffatt, H. K. 1967 On the suppression of turbulence by a uniform magnetic field. $J$. Fluid Mech. 28, 571-592. 
Müller, W.-C. \& CARATi, D. 2002 Dynamic gradient-diffusion subgrid models for incompressible magnetohydrodynamic turbulence. Physics of Plasmas 9 (3), 824834.

Roberts, P. H. 1967 An Introduction to Magnetohydrodynamics. American Elsevier Publishing Company, Inc. New York.

Rogallo, R. 1981 Numerical experiments in homogeneous turbulence. NASA technical Memorandum 81315. NASA, Ames Research Center.

Schumann, U. 1976 Numerical simulation of the transition from three- to twodimensional turbulence under a uniform magnetic field. J. Fluid Mech. 74, 31-58.

ShimomurA, Y. 1991 Large eddy simulation of magnetohydrodynamic turbulent channel flows under a uniform magnetic field. Phys. Fluids A 3 (12), 3098.

Smagorinsky, J. 1963 General circulation experiments with the primitive equations: 1. the basic experiment. Month. Weather Rev. 91, 99-164.

Theobald, M., Fox, P. \& Sofia, S. 1994 A subgrid-scale resistivity for magnetohydrodynamics. Phys. Plasmas 1, 3016.

Yoshizawa, A. 1987 Subgrid modeling for magnetohydrodynamic turbulent shear-flows. Phys. Fluids 30 (4), 1089-1095.

Zhou, Y. \& Vahala, G. 1991 Aspects of subgrid modelling and large-eddy simulation of magnetohydrodynamic turbulence. J. Plasma Physics 45, 239-249. 